spect to $\mathfrak{F}$ and since for a certain large $w$, their number exceeds $(w+1)^{p-2}$, we conclude that, for a certain large $w, \Sigma_{w}^{p}$ does not contain all power products of degree $\rho$ and weight $w$.

Under our assumption, on the other hand, $\Sigma$ would contain all differential polynomials of the form $\left(a_{0} y+a_{1} d y / d x+\ldots\right.$ $\left.+a_{w} d^{w} y / d x^{w}\right)^{\rho}$, where the $a_{i}$ are arbitrary elements of $\mathfrak{F}$. It is easily seen that each power product of degree $\rho$ and weight $w$ is a linear combination of certain of these differential polynomials and hence is in $\Sigma_{w}^{\rho}$. This contradiction shows that for the system $F=y^{3}=0$ no integer $\rho$ exists or, in other words, differential polynomials $G$ exist having arbitrarily large powers not in $\Sigma$.

YALE UNIVERSITY

\title{
NOTE ON THE GALERKIN AND PAPKOVITCH STRESS FUNCTIONS
}

BY R. D. MINDLIN

1. Introduction. H. M. Westergaard* has given a useful interpretation of the Galerkin stress functions $\dagger$ as the components of a vector function satisfying a fourth order equation. From the Galerkin vector, P. F. Papkovitch $\ddagger$ has developed a new solution of the three-dimensional elasticity equations for a homogeneous, isotropic solid in terms of harmonic functions. The same solution has been given by H. Neuber. $\$$

Some interesting aspects of the Galerkin and Papkovitch functions may be observed when they are approached from a consideration of Helmholtz's theorem. In so doing, it is found that these functions may be reached by a direct analytical process and that they are connected through simple functional rela-

* H. M. Westergaard, this Bulletin, vol. 41 (1935), p. 695.

$\dagger$ B. Galerkin, Comptes Rendus, vol. 190 (1930), p. 1047. See also Todhunter and Pearson, History of Elasticity, vol. 2, part 2, pp. 268-270.

‡P. F. Papkovitch, Comptes Rendus, vol. 195 (1932), pp. 513, 754. J. N. Goodler calls attention to Todhunter and Pearson, loc. cit., vol. 2, part 2, p. 373 .

$\S$ H. Neuber, Zeitschrift für angewandte Mathematik und Mechanik, vol. 14 (1934), p. 203. 
tions with each other and with the potentials of the dilatation and rotation.

2. The Helmholtz Transformation. It is known that the displacement which gives rise to the dilatation is the gradient of a scalar potential function and the displacement which produces the rotation is the curl of a solenoidal vector potential function, so that the displacement may be expressed by

$$
\mathbf{o}=\operatorname{grad} \phi+\operatorname{curl} S,
$$

where $\mathbf{\varrho}$ is the vector displacement, $\phi$ is a scalar potential and $\mathbf{S}$ is a vector potential such that $\operatorname{div} S=0$. This transformation is commonly associated with the name of Helmholtz.

3. Equilibrium Equation. In vector form, the equilibrium equation in terms of the displacement is

$$
\Delta \varrho+\frac{1}{1-2 \mu} \operatorname{grad} \operatorname{div} \varrho=-\frac{K}{G},
$$

where $\Delta$ is Laplace's operator, $\mu$ is Poisson's ratio, $K$ is the vector body force per unit of volume, and $G$ is the modulus of rigidity.

Combining equations (1) and (2) and writing

$$
\alpha=\frac{2(1-\mu)}{1-2 \mu}
$$

we obtain the equilibrium equation in terms of the potential functions $\phi$ and $S$ :

$$
\Delta[\alpha \operatorname{grad} \phi+\operatorname{curl} S]=-\frac{K}{G} .
$$

4. The Galerkin Vector. Since $S$ is solenoidal, it may be represented by the curl of a vector function. Let

$$
S=-\operatorname{curl} W \text {. }
$$

The functions $\phi$ and $S$ are independent functions and we may therefore write

$$
\phi=\frac{1}{\alpha} \operatorname{div} W
$$


Substituting equations (4) and (5) in the equilibrium equation (3), we obtain

$$
\Delta[\operatorname{grad} \operatorname{div} W-\operatorname{curl} \operatorname{curl} W]=-\frac{K}{G},
$$

and, making use of the identity

$$
\operatorname{curl} \operatorname{curl}=\operatorname{grad} \operatorname{div}-\Delta,
$$

we obtain

$$
\Delta \Delta W=-\frac{K}{G} .
$$

Substituting equations (4) and (5) in equation (1), and employing the identity given in equation (6), we obtain

$$
\mathbf{\varrho}=\Delta W-\frac{1}{2(1-\mu)} \operatorname{grad} \operatorname{div} W .
$$

The vector function $W$, satisfying equation (7) and producing the displacement according to equation (8), is identical with the Galerkin vector in the form given by Papkovitch. The relation between $W$ and the Galerkin vector is

$$
W=\frac{1-\mu}{G} F \text {. }
$$

5. The Papkovitch Functions. The quantity in parentheses in equation (3) represents a vector function, say $B$. Hence

$$
B=\alpha \operatorname{grad} \phi+\operatorname{curl} S,
$$

and

$$
\operatorname{div} B=\alpha \Delta \phi .
$$

If the body force is taken equal to zero, equations (3) and (9) specify that $B$ is harmonic, so that the complete solution of equation (10) may be written as

$$
\phi=\frac{1}{2 \alpha}(R \cdot B+\beta),
$$

where $R$ is the position vector of a field point referred to the origin and 


$$
\Delta B=0, \quad \Delta \beta=0 .
$$

Equations (1), (9), and (11) yield the expression for the displacement:

$$
\mathbf{0}=B-\frac{1}{4(1-\mu)} \operatorname{grad}(\boldsymbol{R} \cdot \boldsymbol{B}+\boldsymbol{\beta}) .
$$

The functions $B$ and $\beta$, satisfying equations (12) and producing the displacement according to equation (13), are the Papkovitch functions. They bear the following relations to the corresponding functions $\phi_{0}, \phi_{1}, \phi_{2}, \phi_{3}$ found by Neuber:

$$
\begin{aligned}
& B=\frac{2(1-\mu)}{G}\left(i \phi_{1}+j \phi_{2}+k \phi_{3}\right), \\
& \beta=\frac{2(1-\mu)}{G} \phi_{0},
\end{aligned}
$$

where $\boldsymbol{i}, \boldsymbol{j}, \boldsymbol{k}$ are unit orthogonal vectors.

6. Relations Among the Stress Functions. When the body force is zero, the Galerkin, Papkovitch, and potential functions are found to be connected by the following relations. From equations (4), (5), and (9), we have

$$
\begin{aligned}
B & =\Delta W \\
& =\alpha \operatorname{grad} \phi+\operatorname{curl} s ;
\end{aligned}
$$

from equations (5), (11), and (14), we find

$$
\begin{aligned}
\beta & =2 \operatorname{div} W-R \cdot \Delta W \\
& =\alpha\left(2 \phi-R \cdot \operatorname{grad} \phi-\frac{1}{\alpha} R \cdot \operatorname{curl} S\right),
\end{aligned}
$$

and we have already seen that

$$
\begin{aligned}
& S=-\operatorname{curl} W, \\
& \phi=\frac{1}{\alpha} \operatorname{div} W=\frac{1}{\alpha}(R \cdot B+\beta) .
\end{aligned}
$$

Columbia University 